\title{
Resistance spikes and domain wall loops in Ising quantum Hall ferromagnets
}

\author{
T. Jungwirth ${ }^{1,2}$ and A.H. MacDonald ${ }^{1}$ \\ ${ }^{1}$ Department of Physics, The University of Texas, Austin, TX 78712 \\ ${ }^{2}$ Institute of Physics ASCR, Cukrovarnická 10, 16253 Praha 6, Czech Republic
}

(November 5, 2018)

\begin{abstract}
We explain the recent observation of resistance spikes and hysteretic transport properties in Ising quantum Hall ferromagnets in terms of the unique physics of their domain walls. Self-consistent RPA/Hartree-Fock theory is applied to microscopically determine properties of the ground state and domain-wall excitations. In these systems domain wall loops support one-dimensional electron systems with an effective mass comparable to the bare electron mass and may carry charge. Our theory is able to account quantitatively for the experimental Ising critical temperature and to explain characteristics of the resistive hysteresis loops.
\end{abstract}

For two-dimensional electrons in a perpendicular magnetic field $B_{\perp}$, independent electron eigenstates occur in manifolds known as Landau levels with macroscopic degeneracy $A B_{\perp} / \Phi_{0}$, where $A$ is the sample area and $\Phi_{0}$ is the magnetic flux quantum. The zero-width energy bands are responsible for a tremendous variety of many-body physics that has been observed in the quantum Hall regime [1]2]. Quantum Hall ferromagnetism, of interest here, occurs when two different Landau levels distinguished by the cyclotron energy, spin, or quantum well subband labels of their orbitals are brought into energetic alignment and the Landau level filling factor $\nu$ is close to an integer. Neglecting charge fluctuations, low-energy states of quantum Hall ferromagnets (QHFs) are specified by assigning to each orbital in the Landau level a two-component spinor $\left(\cos \theta / 2, e^{i \varphi} \sin \theta / 2\right)$ corresponding to a pseudospin oriented along a general unit vector $\hat{m}=(\sin \theta \cos \varphi, \sin \theta \sin \varphi, \cos \theta)$. (The influence of remote Landau levels can be captured perturbatively as necessary.) While ordered states can occur when any two Landau levels simultaneously approach the chemical potential, the nature of the ground state is sensitive to the microscopic character of the crossing Landau levels [3, 1. Isotropic [5, 6], XY [6] 8] and Ising QHFs [3,9] 12] are now well established. Our work is motivated by the recent observation [13] of hysteretic transport and unexplained resistance spikes when Landau levels with different quantized kinetic (cyclotron) energies cross. We argue that the resistance spikes are due to charge transport in the 1D quasiparticle systems of long domain wall loops and establish a correspondence between their occurrence and vanishing domain-wall free-energy density at the Ising transition temperature, $T_{c}$.

The dependence of the uniform QHF state energy per electron on pseudospin orientation has the form [ [ 1 ]: $E[\hat{m}]=-b m_{z}-J m_{z}^{2}$, where $b$ is an effective magnetic field that includes both single-particle Landau level splitting and interaction contributions 跑 and $J>0$ is an effective Ising interaction parameter. At $b=0$, the $m_{z}=1$ (pseudospin $\uparrow$ ) and $m_{z}=-1$ (pseudospin $\downarrow$ ) states are degenerate. In the following we establish an association between $b=0$ and the experimental resistance spikes, and propose an explanation for the spike origin. For tilted magnetic fields and variable 2D electron densities, the $b=0$ condition at a given filling factor is achieved along a continuous line in the two-dimensional $\left(B_{t o t}-B_{\perp}\right)$ space, which can be explored experimentally by tilting the field away from the sample normal. ( $B_{t o t}$ is the total magnetic field.)

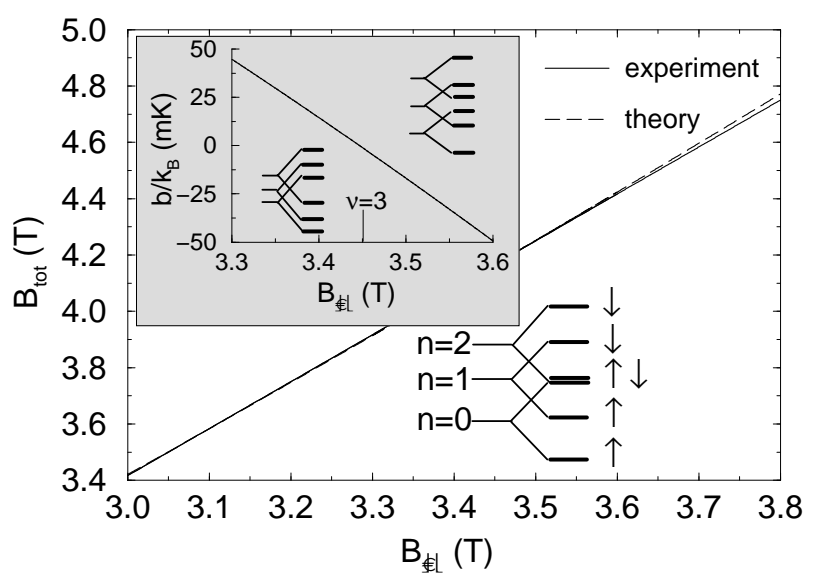

FIG. 1. Main graph: Theoretical (dashed) line of the $n=2$ spin-up and $n=0$ spin-down level coincidences in the perpendicular magnetic field - total field space, calculated using nominal sample parameters of De Poortere et al. [13] and taking into account RPA-screening of the Coulomb interaction. The density of the $2 \mathrm{D}$ electron system is $2.5 \times 10^{11} \mathrm{~cm}^{-2}$. The solid line in the main graph is a fit to the experimental magnetoresistance spike positions presented in Ref. 13] in Fig. 3(A). Inset: Effective external field of the Ising model calculated as a function of the perpendicular magnetic field at a fixed field tilt angle $34^{\circ}$. Landau level configurations at positive and negative values of the $b$-field are also indicated in the inset. 
In Fig. 11 we compare our theoretical [3, 14, $b=0$ line for $\nu=3$, based on numerical self-consistent-field calculations for the geometry of De Poortere et al.'s sample and on many-body RPA/Hartree-Fock theory, with the line along which resistance spikes were observed. The coincidence of these two curves strongly suggests that the spikes occur when $b=0$. The same calculations [4] yield the estimate $J=0.018 e^{2} / \epsilon \ell / k_{B} \sim 2 \mathrm{~K}$, where $\ell$ is the magnetic length defined by $2 \pi \ell^{2} B_{\perp}=\Phi_{0}$.

The ground state of an Ising QHF has $m_{z}=1$ for $b>0$ and $m_{z}=-1$ for $b<0$. At finite temperatures, non-trivial pseudospin magnetization configurations become important. For Ising QHFs, an elementary calculation shows that spin-wave collective excitations have a gap $\Delta / k_{B}=4 J / k_{B} \sim 8 \mathrm{~K}$. Since the hysteretic resistance spikes occur only for $T<0.5 \mathrm{~K}$, spin-wave excitations cannot play a role. Instead, as we now explain, the important thermal fluctuations in Ising QHFs involve domain walls between $m_{z}=1$ and $m_{z}=-1$ regions of the sample.

In classical 2D Ising models the critical temperature can be understood as a competition between unfavorable near-neighbor-spin interaction energy along a domain wall, $L \gamma$, and the wall configurational entropy, $L s_{c}=L / \xi k_{B} \ln (3)$, where $\xi$ is the domain wall persistence length. Both give free-energy contributions proportional to wall length, $L$, with the former effect favoring short walls and the latter contribution, which is proportional to temperature, favoring long walls. For $T>T_{c}$ the system free energy is lowered when domain walls expand to the sample perimeters, destroying magnetic order. The structure of domain walls is more complicated in Ising QHFs. As the domain wall is transversed, the local pseudospin orientation goes from the north pole $\left(m_{z}=1\right)$ to the south pole $\left(m_{z}=-1\right)$, at a fixed orientation $\varphi$ of its $\hat{x}-\hat{y}$ plane projection. We have evaluated the energy per unit length $\gamma$ of an infinite domain wall by solving self-consistent Hartree-Fock equations:

$$
\tan \theta(X)=\frac{-2\left(b-H_{\uparrow, \downarrow}^{F}(X)\right)}{H_{\uparrow, \uparrow}^{H}(X)+H_{\uparrow, \uparrow}^{F}(X)-H_{\downarrow, \downarrow}^{H}(X)-H_{\downarrow, \downarrow}^{F}(X)},
$$

where the Hartree energy is given by

$$
\begin{aligned}
& H_{\sigma, \sigma^{\prime}}^{H}(X)=\sum_{X^{\prime}} \int d^{3} \vec{r}_{1} \int d^{3} \vec{r}_{2} V\left(\vec{r}_{1}-\vec{r}_{2}\right) \times \\
& \psi_{\sigma, X}^{*}\left(\vec{r}_{1}\right) \psi_{\sigma^{\prime}, X}\left(\vec{r}_{1}\right) \psi_{\hat{m}\left(X^{\prime}\right), X^{\prime}}^{*}\left(\vec{r}_{2}\right) \psi_{\hat{m}\left(X^{\prime}\right), X^{\prime}}\left(\vec{r}_{2}\right)
\end{aligned}
$$

and the exchange energy by

$$
\begin{aligned}
& H_{\sigma, \sigma^{\prime}}^{F}(X)=-\sum_{X^{\prime}} \int d^{3} \vec{r}_{1} \int d^{3} \vec{r}_{2} V\left(\vec{r}_{1}-\vec{r}_{2}\right) \times \\
& \psi_{\sigma, X}^{*}\left(\vec{r}_{1}\right) \psi_{\sigma^{\prime}, X}\left(\vec{r}_{2}\right) \psi_{\hat{m}\left(X^{\prime}\right), X^{\prime}}^{*}\left(\vec{r}_{1}\right) \psi_{\hat{m}\left(X^{\prime}\right), X^{\prime}}\left(\vec{r}_{2}\right) .
\end{aligned}
$$

In Eqs. (2) and (3), $V\left(\vec{r}_{1}-\vec{r}_{2}\right)$ is the RPA-screened Coulomb potential and the self-consistent-field oneparticle orbitals, $\psi_{\sigma, X}(\vec{r})$, are extended along the domain wall and localized near wavevector $k$ dependent guiding centers $X=k \ell^{2}$. The energy density $\gamma$ is proportional to the increase in Hartree-Fock quasiparticle energies, integrated across the domain wall. We find that the domain wall width is typically several magnetic lengths and for the $\nu=3$ coincidence we find that $\gamma \ell=0.009 e^{2} / \epsilon \ell$.

A unique property of QHFs is the proportionality between electron charge density and pseudospin topological index density. It is this property that is responsible for the fascinating skyrmion physics extensively studied in the isotropic case [5,6]. In the case of Ising QHFs, the proportionality implies a local excess charge per unit length along a domain wall $\rho_{\|}=e \nabla \varphi \cdot \hat{n} /(2 \pi)$ where $\hat{n}$ specifies the local direction along the domain wall. Single-valuedness of the magnetization requires that the winding number of the angle $\varphi$ around a domain wall loop be quantized in units of $2 \pi$ and hence that the excess charge of a domain wall loop be quantized in units of the the electron charge $e$. The free-energy associated with the classical $\varphi$ field fluctuations within a domain wall,

$$
f_{\varphi}=\frac{1}{L} k_{B} T \ln Z ; Z=\int \mathcal{D} \varphi \exp \left(E_{c}[\varphi] / k_{B} T\right),
$$

is controlled by the Coulomb interaction energy $E_{c}$ due to the consequent charge fluctuations.

Assuming a domain wall persistence length $\xi \approx \ell$, the free energy density of Ising QHF domain wall loops is given by $f=k_{B} T \ln (3) / \ell+\gamma+f_{\varphi}$ and equals zero at $T=T_{c}$. For the $\nu=3 \mathrm{QHF}$, these considerations imply that infinitely long domain walls proliferate and order is lost for $T$ larger than the transition temperature $T_{c} \approx 500 \mathrm{mK}$. The close correspondence between this $T_{c}$ estimate, and the maximum temperature $(430 \mathrm{mK})$ at which hysteretic resistance spikes are observed 13. strongly supports our contention that the unusual transport phenomena are a consequence of the existence of long domain wall loops in these materials. In the following we first discuss the $b$-field, Landau level filling factor, and temperature dependence of the system's domain-wall soup and then demonstrate that this picture can account for many details of the transport observations.

Domain wall loops are characterized by their length and by their charge, with infinitely long loops appearing only for $b=0$ and $T>T_{c}$. For finite size loops with a typical radius larger than the domain wall width we can use our Hartree-Fock self-consistent results for $\theta(X)$ to estimate the Coulomb self-interaction energy. A twodimensional charge density of a circular loop with excess charge $e$ distributed uniformly along the domain wall is given by

$$
\rho_{2 D}(r)=\frac{e}{4 \pi r} \frac{d}{d r} \cos \theta(r)
$$


Since the corresponding Coulomb self-interaction energy is proportional to the square of the charge and approximately inversely proportional to the length, charged domain wall loops have a higher energy and, at integer filling factors, will always be less common than neutral domain wall loops. Resistance spikes are generically observed slightly away from integer filling factors, however, and here the situation changes because the domain wall loops can exchange charge with the rest of the $2 \mathrm{D}$ electron system.

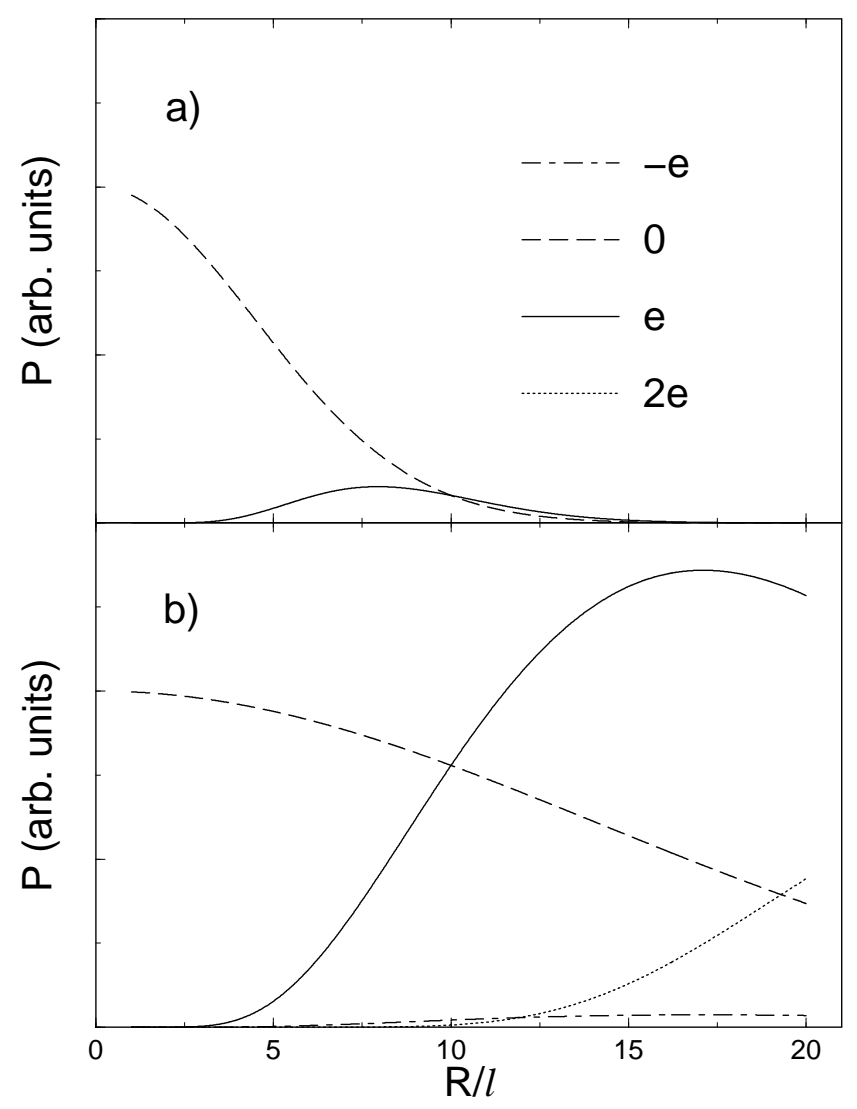

FIG. 2. Near $-T_{c}, \nu>3$, statistical weights of domain wall loops with excess charge from $-e$ to $2 e$ are plotted as a function of the loop radius. a) For $b / k_{B} \approx 20 \mathrm{mK}$, the effective external field corresponds to the edge of the measured resistance spike. Small neutral domain wall loops dominate in this case. b) For $b \approx 0$, the coincidence condition is approached corresponding to the spike maximum. The typical domain wall loops are large and carry an excess charge.

The lowest energy elementary charged excitations of the $\nu=3$ ground state are ordinary Hartree-Fock electron and hole quasiparticles [17], not charged domain wall loops. In systems with no disorder, the chemical potential lies in the middle of the Hartree-Fock gap when $\nu$ is an integer but moves quickly (by $\delta \mu$ ) toward the electron quasiparticle energy for $\nu>3$ and toward the hole quasiparticle energy for $\nu<3$. These chemical potential shifts will be reduced by disorder which broadens the quasiparticle bands. The change in chemical potential favors charge $Q$ over neutral skyrmions by a large factor $\exp \left(Q|\delta \mu| \mid / k_{B} T\right)$. This factor can be estimated quantitatively using the experimental value of the quasiparticle excitation gap $(\sim 2 \mathrm{~K})$ 13].

Also important in controlling the domain wall soup is the effective field $b$, which measures the distance from Landau level coincidence. For $b \neq 0$ the energy of a domain wall loop has a contribution proportional to $b$ and the number of condensate electrons contained within the loop:

$$
E_{b}=-\frac{b}{2 \ell^{2}} \int d r r[\cos \theta(r)-1] .
$$

This contribution will decrease the number of large domain wall loops enclosing the minority phase and is independent of the charge carried by the loop. Summing up $E_{b}$, the Coulomb self-interaction and chemical potential contributions and the Hartree-Fock domain wall energy of a circular loop of radius $R, 2 \pi R \gamma$, we can estimate statistical weights of neutral and charged domain wall loops in the sample of De Poortere et al. In Fig. 2 we plot our results for temperature near $T_{c}$ and $\nu>3$. For non-zero $b$-fields, small neutral domain wall loops dominate, while typical loops at the coincidence are large and carry an excess charge.

We now address characteristic features of the measured resistive hysteresis loop. Dissipation can occur in Ising QHFs as a result of Hartree-Fock quasiparticle diffusion, charged domain-wall-loop diffusion, or as a result of charge diffusion within domain-wall loops. It is clear that the resistance spikes, which appear only for small $b$ and $T<T_{c}$, are associated with the appearance in the sample of large domain-wall loops. Even though these loops tend to be charged at the spike maximum, we expect that they will be immobile because of their large size and that dissipation due to their motion is small. Instead we propose that mobile quasiparticles inside domain walls are responsible for the increase of dissipation. In Fig. 3 we plot the Hartree-Fock quasiparticle energies in a crosssection of a domain wall, obtained from Eqs. (1)-(3). In the center of the domain wall the quasiparticle gap is reduced by nearly $50 \%$. Away from integer filling factors, the bottom of these 1D quasiparticle bands will lie below the chemical potential which is pinned to the bulk quasiparticle energies. We note that, unlike quantum Hall edge states, counter propagating states exist within each loop. At $\nu \approx 3$, for example, the $1 \mathrm{D}$ states have a nearly parabolic dispersion characterized by an effective mass $m^{*} \approx 2 m_{e}$. The particles can cross the sample by scattering between overlapping loops. For $\nu=3$ and $T \approx T_{c}$, it follows from inset of Fig. 1 and from Fig. 2 that the characteristic loop radius at the spike edge is $3 \ell$. At low temperatures or small magnetic lengths (high 2D electron gas densities), domain wall loops become small and dilute, loops do not overlap, and charge diffusion within 
domain walls cannot contribute to dissipation. This explains the absence of resistance spikes at Landau level coincidence [13] under these circumstances.

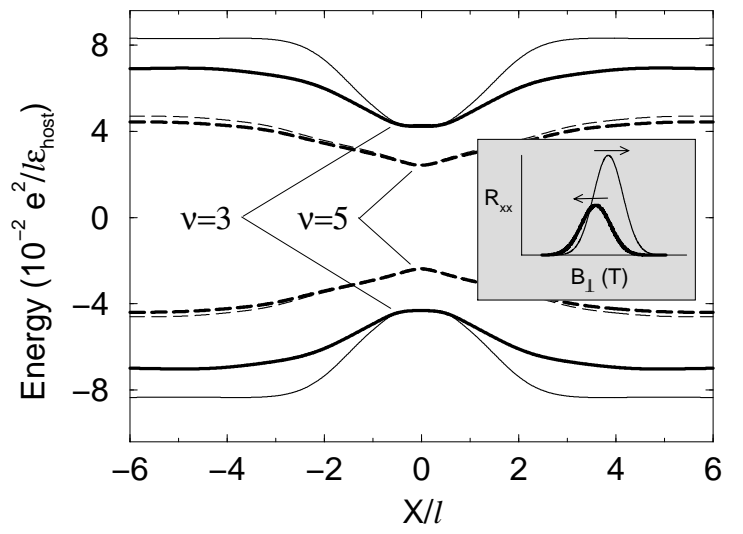

FIG. 3. Hartree-Fock quasiparticle energies, measured from the integer filling factor chemical potential, are plotted as a function of orbit guiding center for zero effective external field $b$. For $|X| \gg 0$ the spectrum describes ordinary, bulk electron and hole quasiparticles. Near the domain wall center a parabolicly dispersing 1D channel is formed. The thin and thick lines are obtained assuming pseudospin-up and pseudospin-down majority polarizations, respectively. At $\nu=3$ (solid lines), the screening of the Coulomb interaction is substantially stronger in the latter case resulting in different quasiparticle spectra for the two configurations. The inset illustrates schematically the corresponding peak asymmetry in the measured magnetoresistance near filling factor $\nu=3$. For comparison, we also show in the main graph spectra for $\nu=5$ (dashed lines). The negligible difference between pseudospin-up and pseudospin-down majority polarizations is consistent with the similar peak heights recorded in up and down magnetic field sweeps near $\nu=5$.

The above mechanism also explains the different resistance spike heights observed in up and down field sweeps [13] near $\nu=3$. As shown in the inset of Fig. 1, the majority pseudospin Landau level for the up-sweep is the $n=2$ spin-up level while for the down-sweep it is the $n=0$ spin-down Landau level. This difference in Landau level configurations in the two sweep directions alters remote Landau level screening in the sample which has a marked effect on the quasiparticle energy spectrum. The reduction of the quasiparticle gap in the domain wall, relative to its bulk value, is stronger in the up-sweep case, leading to more domain-wall quasiparticles and more dissipation, as seen in experiment. Similar agreement between the measured hysteresis loop properties and domain wall quasiparticle spectra applies for $\nu=4$ [16]. In Fig. 3 we also plot energy spectra at $\nu=5$ which are nearly identical for the up or down majority pseudospin orientations. This explains the absence of peak-height asymmetry in the hysteresis measurement at this filling factor $\nu=5$. 16 .

We thank Etienne De Poortere, Herbert Fertig, and Mansour Shayegan for many important discussions. Our work was supported by R.A. Welch Foundation, by Minsistry of Education of the Czech Republic under Grant OC P5.10, and by the Grant Agency of the Czech Republic under Grant 202/01/0754.

[1] R.E. Prange and S.M. Girvin (eds), The Quantum Hall Effect (Springer, New York, 1990).

[2] S. Das Sarma and A. Pinczuk (eds), Perspectives in Quantum Hall Effects (Wiley, New York, 1996).

[3] T. Jungwirth, S.P. Shukla, L. Smrčka, M. Shayegan, and A.H. MacDonald, Phys. Rev. Lett. 81, 2328 (1998).

[4] T. Jungwirth and A.H. MacDonald, Phys. Rev. B 63, 035305 (2001).

[5] R. Tycko, S.E. Barrett, G. Dabbagh, L.N. Pfeiffer, and K.W. West, Science 268, 1460 (1995).

[6] S.M. Girvin and A.H. MacDonald, in Ref. [2].

[7] J.P. Eisenstein, in Reference (5).

[8] I.B. Spielman, J.P. Eisenstein, L.N. Pfeiffer, and K.W. West, Phys. Rev. Lett. 84, 5808 (2000).

[9] V. Piazza, V. Pellegrini, F. Beltram, W. Wegscheider, T. Jungwirth, and A.H. MacDonald, Nature 402, 638 (1999).

[10] J. Eom, H. Cho, W. Kang, K.L. Campman, A.C. Gossard, M. Bichler, and W. Wegscheider, Science 289, 2320 (2000).

[11] G.F. Giuliani and J.J. Quinn, Phys. Rev. B 31, 6228 (1985).

[12] A.J. Daneshvar, C.J.B. Ford, M.Y. Simmons, A.V. Kahetskii, A.R. Hamilton, M. Pepper, and D.A. Ritchie, Phys. Rev. Lett. 79, 4449 (1997).

[13] E. P. De Poortere, E. Tutuc, S. J. Papadakis, and M. Shayegan, Science 290, 1546 (2000).

[14] T. Jungwirth, A.H. MacDonald, L. Smrčka, and S.M. Girvin, Phys. Rev. B 60, 15574 (1999).

[15] Self-consistent local-spin-density approximation calculations were carried out to establish that for the $15 \mathrm{~nm}$ wide AlAs quantum wells studied in these experiments, orbital effects of the in-plane field are negligibly small. In the calculation we assumed Landé g-factor $g=1.9$, electron effective mass $m^{*}=0.41 m_{e}$, and average heterostructure dielectric constant $\epsilon=11.5$.

[16] E. P. De Poortere, E. Tutuc, S. J. Papadakis, and M. Shayegan, unpublished data.

[17] D. Lilliehöök, Phys. Rev. B 62, 7303 (2000). 